\title{
Intake of B vitamins in UK dwelling South Asian and White Caucasian women: Results from the D-FINES study
}

\author{
Andrea Darling, Kourosh Ahmadi and Susan Lanham-New \\ University of Surrey, Guildford, United Kingdom
}

\begin{abstract}
Adequate intakes of the B vitamins are essential for health; however there is a lack of data concerning B vitamin intakes in UK dwelling South Asian (SA) groups. We aimed to investigate whether UK SA women meet the LRNI for B vitamins, and whether their intake differs from same-age White Caucasian (WC) women. We used summer 2006 dietary intake data from the Food Standards Agency (FSA) funded D-FINES study (Vitamin D, Food Intake, Nutrition and Exposure to Sunlight in Southern England, project N05064). After removal of over- and under-reporters (energy: BMR ratio $<1$ or $>1.6$ ) there were $n=29 \mathrm{SA}$ and $\mathrm{n}=146 \mathrm{WC}$ subjects. The two groups did not differ significantly in age and BMI. Overall mean (SD) for age was 50.6 (13.6) years and for BMI was 26.8 (4.8). In SA, 41\% were Bangladeshi or Pakistani, 28\% were Indian and 31\% were of other ethnicity. Independent T-tests, using log transformed data, showed no statistically significant differences for any B vitamin (Bonferroni revised $\mathrm{p}$ value: $<0.008)$. Results were as follows, giving median (IQR): Thiamine (mg) $1.5(0.5) \mathrm{SA}$ vs $1.4(0.5) \mathrm{WC}$; $(\mathrm{P}=0.8)$; Riboflavin (mg) 1.3 (0.5) SA vs. 1.5 (0.6) WC $(\mathrm{P}=0.08)$; Niacin $(\mathrm{mg}) 30.7$ (13.7) SA vs $33.3(9.8) \mathrm{WC}(\mathrm{P}=0.4) ; \mathrm{B}_{6}(\mathrm{mg}) 1.7(0.5) \mathrm{SA}$ vs $1.9(0.7) \mathrm{WC}(\mathrm{P}=0.2) ; \mathrm{B}_{12}(\mathrm{micrograms}) 2.8$ (0.05) SA vs $3.6(2.5) \mathrm{WC}(\mathrm{P}=0.02)$; Folate (micrograms) 213 (93) SA vs 231 (82) WC $(\mathrm{P}=0.8)$. In terms of percentages below the LRNI: Thiamine $0 \%$ SA and $0.7 \%(n=1) \mathrm{WC}$; Riboflavin $0 \%$ SA and $1.4 \%(n=2)$ WC; $\mathrm{B}_{12} 10 \%(\mathrm{n}=3) \mathrm{SA}$ and $0 \% \mathrm{WC}$. For Niacin, $\mathrm{B}_{6}$ and Folate no women in either group were below the LRNI. Overall, there were no ethnic differences in $\mathrm{B}$ vitamin intake by ethnicity. There was a trend for a slightly lower $\mathrm{B}_{12}$ intake in SA but this did not reach statistical significance after adjustment for multiple testing. It is of concern that $10 \%$ of SA did not meet the LRNI for $\mathrm{B}_{12}$. Of this $10 \%$, the majority were not vegetarian or vegan. The sample size for SA was very small and further research is now required in a larger sample to confirm this finding. The D-FINES study was funded by the UK FSA (N05064). The views expressed are those of the authors alone.
\end{abstract}

\section{Conflict of Interest}

There is no conflict of interest 